\title{
Coherent and Squeezed States: Introductory Review of Basic Notions, Properties, and Generalizations
}

\author{
Oscar Rosas-Ortiz
}

To Prof. Veronique Hussin on his 60th birthday with friendship and scientific admiration.

\begin{abstract}
A short review of the main properties of coherent and squeezed states is given in the introductory form. The efforts are addressed to clarify concepts and notions, including some passages of the history of science, with the aim of facilitating the subject for nonspecialists. In this sense, the present work is intended to be complementary to other papers of the same nature and subject in current circulation.
\end{abstract}

Keywords Coherent states - Squeezed states - Nonclassical states · Optical detection · Optical coherence · Wave packets · Minimum uncertainty · Harmonic oscillator · Riccati equation · Ermakov equation

\section{Introduction}

Optical coherence refers to the correlation between the fluctuations at different space-time points in a given electromagnetic field. The related phenomena are described in statistical form by necessity, and include interference as the simplest case in which correlations between light beams are revealed [1]. Until the first half of the last century the classification of coherence was somehow based on the averaged intensity of field superpositions. Indeed, with the usual conditions of stationarity and ergodicity, the familiar concept of coherence is associated with the sinusoidal modulation of the averaged intensity that arises when two fields are superposed. Such a modulation produces the extremal values $\left\langle I_{m a x}\right\rangle_{a v}$ and $\left\langle I_{m i n}\right\rangle_{a v}$, which are used to define the visibility of interference fringes

O. Rosas-Ortiz ( $\square)$

Physics Department, Cinvestav, México City, Mexico

e-mail: orosas@ fis.cinvestav.mx 